\title{
RÉCORDS OLÍMPICOS: HUMANOS VS INSECTOS
}

\section{OLYMPIC RECORDS: HUMAN VS INSECTS}

\author{
Ingeborg Zenner de Polanía1 \\ 1I.A., M.Sc., Ph.D. Entomóloga. Universidad de Ciencias Aplicadas y Ambientales U.D.C.A, Calle 222 No.55-37, Bogotá, D.C. \\ izenner@udca.edu.co
}

Rev. U.D.C.A Act. \& Div. Cient. 15 (Supl. Olimpismo): 37 - 45, 2012

\section{RESUMEN}

Basado en observaciones, en mediciones y en literatura acerca de las habilidades deportivas de los insectos, en los tres deportes reconocidos de los Juegos Olímpicos de Verano, el atletismo, la gimnasia y la natación, se comparan algunas marcas deportivas, recientemente establecidas por hombres y por mujeres con aquellos que, determinados insectos, sostienen desde hace millones de años. Estos artrópodos, si compitiesen con los humanos, ganarían en la mayoría de las justas, en las que, por sus características, podrían participar, ya sea a nivel mundial o en juegos olímpicos. En el levantamiento de pesas, en cualquiera de las modalidades, compiten los escarabajos peloteros y las hormigas con los insectos; ganarían los cucarrones, reconocidos también como los insectos sagrados de los antiguos egipcios. Disputa con ellos la medalla, el escarabajo hércules o rinoceronte, Dynastes hercules, cucarrón emblemático de las selvas tropicales de Suramérica, incluyendo a Colombia. Las chinches acuáticas de las familias Notonectidae y Corixidae son nadadores por excelencia de dos estilos, espalda y pecho y no es la pulga, que se pensaba sostenía el récord mundial de salto alto y largo, sino un insecto salivazo o mión (Cercopidae), plaga de alfalfa. Igualmente, y como ocurre casi siempre en los juegos olímpicos, donde alguien le quita el récord a un atleta, hace poco, otro insecto le quitó la marca de corredor más rápido a la cucaracha americana. Afortunadamente, los humanos no compiten en deportes con los insectos, pero sí luchan con algunos de ellos, con éxito limitado, principalmente, por alimentos.

Palabras clave: Competencias deportivas, olimpíadas de verano, marcas, cucaracha, pulga.

\section{SUMMARY}

Based on observations, measurements and literature on the sport abilities of insects within the three recognized catego- ries of the Olympic Summer Games, athletics, gymnastics and swimming, some sporting marks, recently established by men and women, are compared with those, which specific insects sustain since millions of years. If these arthropods would compete with athletes, they would gain all competences, in which their characteristics would permit their participation, in both world and Olympic Game. In weight lifting, within any category, dung beetles and ants would compete, winning the gold medals the first group, to which the sacred dung beetles of the ancient Egyptians belong. They will be challenged by the rhinoceros beetle, Dynastes hercules, emblematic scarab of the South-American rainforests, including Colombia. The aquatic bugs of the families Notonectidae and Corixidae are swimmers by excellence of both styles, back and breast and, it is not the flea which sustains the world record of high and large jumps, but a spittlebug or froghopper (Cercopidae), an insect pest of alfalfa. Equally, and as it happens during the Olympic Games, where somebody takes a record away from an athlete, an other insect beats the score of the American cockroach as fastest runner. Fortunately, humans do not compete in games with insects, but do so, although with limited extent, with some of them for food.

Key words: Sport competence, summer Olympic Games, scores, cockroach, flea.

\section{INTRODUCCIÓN}

Desde la edad de piedra, los humanos se tenían que mover corriendo, saltando o nadando, escapando de las fieras salvajes, persiguiéndolas para lograr el alimento y las pieles para sus vestidos, compitiendo entre sí y con los animales. Menciona McComb (1999), que las competencias atléticas pueden ser tan viejas como los humanos mismos y que, a través del tiempo, las contiendas han tenido influencias positivas y negativas en, prácticamente, todas las facetas de la 
vida. Igualmente, indica que debemos diferenciar entre los deportes profesionales y amateurs. ¿A cuál de las dos modalidades pertenecen los insectos?

Se puede sugerir que el deporte nació en las diferentes civilizaciones, primero, por demostrar las habilidades en la caza y en el combate; posteriormente, hombres y mujeres, por su vida cada vez más sedentaria, buscaban actividades al aire libre, originalmente para su diversión y, después para competir y ganar o perder. En cambio, los insectos, que aparecieron mucho antes que el Homo sapiens, se tienen vestigios de su existencia desde el carbonífero, es decir, hace más de 400 millones de años, era de la cual se conocen fósiles de insectos (Engel \& Grimaldi, 2004), ya practicaban el salto largo, corrían y nadaban y ejercían otros actividades para defenderse, para sobrevivir o para alcanzar su presa.

Previo a los juegos olímpicos antiguos (776 a.C.), no tanto como sana competenciay diversión, sino más bien como eventos rituales, antes y después de sacrificios humanos, los mayas y los aztecas practicaban los juegos de pelota, durante más de tres mil años de la prehistoria precolombina mesoamericana. Estos juegos están bien documentados (Taladoire, 2000) y muestran entre sus símbolos a mariposas (Insecta: Lepidoptera), que son un motivo frecuente en el Tlalocan de los murales de Tepantitla, como lo sostiene Uriarte (2000).

El lanzamiento de jabalina, actualmente deporte olímpico, fue practicado por los chinos y los egipcios, por el año 4000 a.C., como se puede observar en pinturas y en otros hallazgos arqueológicos; aparentemente, se ejerció lanzando la jabalina a un blanco colocado a variadas distancias (Wikilibro, s.f.); sin embargo, fue más bien un arma de guerra y su uso un mecanismo de defensa y de supervivencia para procurar la alimentación, que consistía en venados y en peces.
Aunque no se puede comparar de manera exacta, algunos insectos de la familia Carabidae (Coleoptera), los escarabajos bombarderos o escopeteros, podrían combatir con los humanos. No usan artefactos, sino glándulas en que almacenan substancias que, al ser amenazados o molestados, disparan una substancia caliente (Eisner et al. 2000), que se podría comparar con una jabalina, bala u honda. Estos insectos solo miden entre 2 y $30 \mathrm{~mm}$ y pueden apuntar hacia su enemigo, un posible depredador o su presa, que puede ser hasta un pequeño sapo, a una distancia hasta de $20 \mathrm{~cm}$, con alta precisión en segundos (Softpedia, 2011; Armitage \& Mullisen, 2003). Dean et al. (1990) al estudiar este mecanismo de defensa determinaron la velocidad que lograba el cucarrón escopetero y encontraron que la rociada o chorro estaba entre los 325 a $1950 \mathrm{~cm} / \mathrm{s}$. Analizando la equivalencia de tamaño, de distancia y de velocidad, ningún ser humano podría disparar una jabalina o una flecha para ganarle al pequeño insecto (Fotografía 1a). El récord del hombre, de estatura de 1,85m, corresponde a una distancia de 90,17m (Jan Zelezny, Sydney 2000), mientras que el insecto que mide hasta $30 \mathrm{~mm}$, alcanza los $20 \mathrm{~cm}$ de distancia.

Otro insecto que se caracteriza por la rapidez y por los movimientos que ejecuta, aunque no compite con los humanos por la captura de presas, es la rezandera Mantis religiosa L. 1758 (Mantodea: Mantidae) (Fotografía 1b). Esta actividad ya no se incluye en los juegos olímpicos, por fortuna, pero en la era romana los gladiadores tenían un arma en forma de red que les permitía capturar al oponente y, con el cual, se podría comparar a la mantis Ésta aguarda una víctima, apoyada en sus patas intermedias y traseras, con las patas delanteras levantadas, con las que atrapa sus presas; otros insectos voladores, como por ejemplo, una mosca, con tal rapidez que, sólo se puede observar antes de que la agarra y, luego, cuando el insecto ya está apresado (Anónimo, 1997).

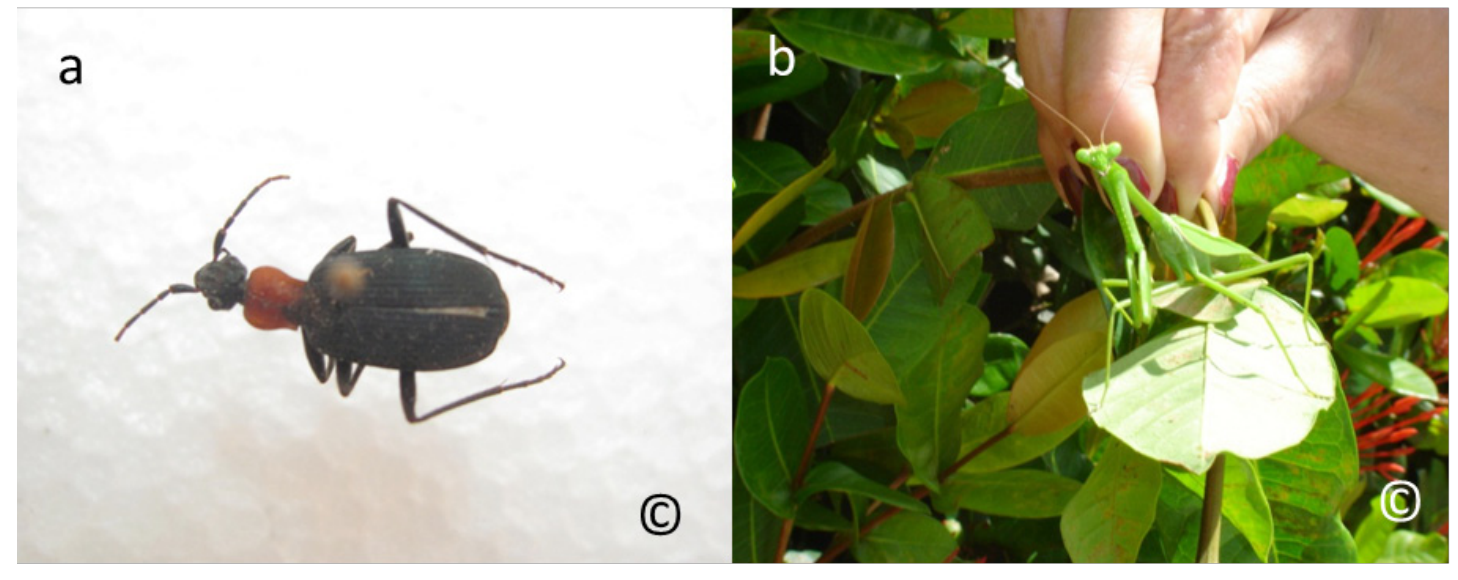

Fotografía 1: a) Escarabajo bombardero o escopetero, Brachinus sp. (Coleoptera: Carabidae). Fotografía: Ing. Agr. Héctor William Duarte; b) Rezandera Mantis religiosa (Mantodea: Mantidae) (fotografía: autora). 
Los insectos no solamente podrían rivalizar con los humanos en competencias deportivas, sino también servir como modelo para estilos deportivos de combate. Tal vez, el ejemplo más conocido, basado en una fábula, hace referencia, precisamente, a la $M$. religiosa y las artes marciales, que celebran sus propias "Olimpiadas de Artes Marciales". Cuenta la leyenda que un día, el Sr. Wang Lang, un artista marcial, contempló el combate entre una rezandera y una chicharra, siendo la ganadora la primera. El artista, se fijó detenidamente en los movimientos de ataque y de defensa de la ganadora, pensando en la posibilidad de crear un estilo que imitaría la actividad del insecto. Capturó el animalito y lo hizo pelear con otros insectos, siguiendo con sus observaciones y "así fue copiando y desarrollándolo con el agregado de técnicas de agarre, ataques explosivos, cortes, deslizamientos e inmovilizaciones un estilo de lucha, que llegaría ha convertirse en el famoso Boxeo de la Mantis o "Tanglangquan" (Arnedo, s.f.).

Los estudios científicos acerca de los insectos y sus habilidades, como corredores, saltadores, nadadores, luchadores y posibles practicantes de otros deportes olímpicos son escasos, lo que obliga a recurrir a la literatura gris que, no necesariamente, se basa en experimentos. Este hecho motivó la realización de una revisión de literatura sobre las destrezas deportivas de determinados insectos, presentes o con parientes en nuestro medio y a la ejecución de unos pocos ensayos, para medir, precisamente, sus capacidades deportivas.

\section{MATERIALES Y MÉTODOS}

Basado en los conocimientos acerca de la biología y los hábitos de algunos insectos, su comportamiento como plaga o benéfico, observaciones de campo y la literatura revisada acerca de sus habilidades de supervivencia o los comentarios escuchados o películas vistas en televisión, se escogieron la natación, el atletismo, el levantamiento de pesas y la lucha libre, como puntos focales de comparación en este estudio.

Como nadadores, se eligieron a dos chinches acuáticos hemípteros, de las familias Notonectidae y Corixidae, comunes en estanques, lagunas u otros depósitos de agua dulce, prácticamente, en todos los pisos térmicos del país. En un estanque de agua lluvias, con crecimiento de algas y larvas de mosquitos y otros insectos, de $2 \mathrm{~m}$ de ancho, $3 \mathrm{~m}$ de largo y $1,50 \mathrm{~m}$ de profundidad, se observaron y se tomaron el tiempo y la distancia nadada, con cronómetro y metro, en varias ocasiones, de la rapidez de desplazamiento, estilo espalda, de 20 adultos de Notonecta sp. (Hemiptera: Notonectidae). Los individuos de esta especie permanecen quietos en el agua, con las patas más o menos extendidas, nadando sobre la espalda rápidamente cuando son molestados o cuando perciben una presa; esta conducta permitió tomar el tiempo de deslizamiento con cierta exactitud.

El nadador escogido, estilo pecho o brazo, fue otra chinche acuática de la familia Corixidae, género cerca a Neosigara, habitante del mismo estanque, insectos parecidos, a simple vista, a los notonéctidos, pero con el cuerpo más aplanado y el hábito de nadar sobre el pecho y no en línea recta, sino en forma de zigzag, lo que dificultó notablemente la toma de datos y medir sus destrezas. Igualmente, se trató de calcular la distancia recorrida y el tiempo gastado, de 20 individuos de cada especie.

Atletismo: no se dispone datos propios, ni se realizaron observaciones de determinados insectos, solamente, se tomaron de la literatura las mediciones de algunos artrópodos que sí existen en el país. Se procura entusiasmar a entomólogos a verificar, científicamente, los datos encontrados, ante todo, aquellos detallados en la literatura gris. Se encontraron mediciones del chapulín o saltamontes (Orthoptera: Tettigoniidae), de pulgas y de un Cercopidae (Hemiptera).

Levantamiento de pesas: Para fines de comparación con datos de artículos publicados, se tomó como ejemplo a la hormiga cortadora de hojas u hormiga arriera, Atta spp. (Hymenoptera: Formicidae, Myrmicinae). Para conocer la carga aproximada que las obreras podrían soportar desde el sitio donde cortaban los pedazos de hoja hasta el nido, se pesaron, de cítricos 44 y de Eugenia sp. (Myrtacea) 45 pedazos de hoja, con una balanza de precisión Ohaus, modelo Adventurer. El peso de las obreras, se obtuvo de la literatura.

Lucha libre: Aunque no se puede comparar este estilo deportivo de los humanos con la realización de lucha entre los diversos insectos, se detalla la lucha de dos géneros de hormigas, basado en observaciones de campo.

Los récords logrados por los campeones olímpicos humanos se consiguieron, para fines de comparación, de páginas de internet (Natación, 2008; Marca (Deporte), 2009; Récords Olímpicos, 2004).

Las fotografías que acompañan el texto, se obtuvieron de insectos existentes en la Colección Entomológica de la Facultad de Ingeniería Agronómica de la Universidad de Ciencias Aplicadas y Ambientales U.D.C.A o en el hábitat natural de estos artrópodos.

\section{RESULTADOS Y DISCUSIÓN}

Natación: En la tabla 1, se consignan los resultados de las mediciones logradas, tanto para Notonecta sp. (Fotografía 2a) como para pos. Neosigara. Los datos no deben ser 
considerados definitivos sino de referencia, ya que los movimientos erráticos de ambos insectos dificultan cronometrar el tiempo gastado en recorrer determinada distancia, pero muestran marcas asombrosas.

Tabla 1. Distancia (cm) y velocidad (seg.), media de la campeona de nado, estilo espalda, Notonecta sp. y de la campeona de brazo, género cerca a Neosigara respectivamente Notonectidae y Corixidae del orden Hemiptera.

\begin{tabular}{|l|c|c|c|}
\hline & cm \pm D.S. & seg. \pm D.S. & cm/seg \pm D.S. \\
\hline Notonecta sp. & $\begin{array}{c}49,11 \pm 8,20 \\
(32,5-61)\end{array}$ & $\begin{array}{c}0,945 \pm 0,16 \\
(0,7-1,3)\end{array}$ & $\begin{array}{c}52,16 \pm 4,63 \\
(43,46-60)\end{array}$ \\
\hline cerca a Neosigara & $37,15 \pm 6,31$ & $0,49 \pm 6,31$ & $75,72 \pm 21,19$ \\
& $(29-51)$ & $(0,3-0,7)$ & $(48,33-123,33)$ \\
\hline
\end{tabular}

Datos entre paréntesis indican el rango.

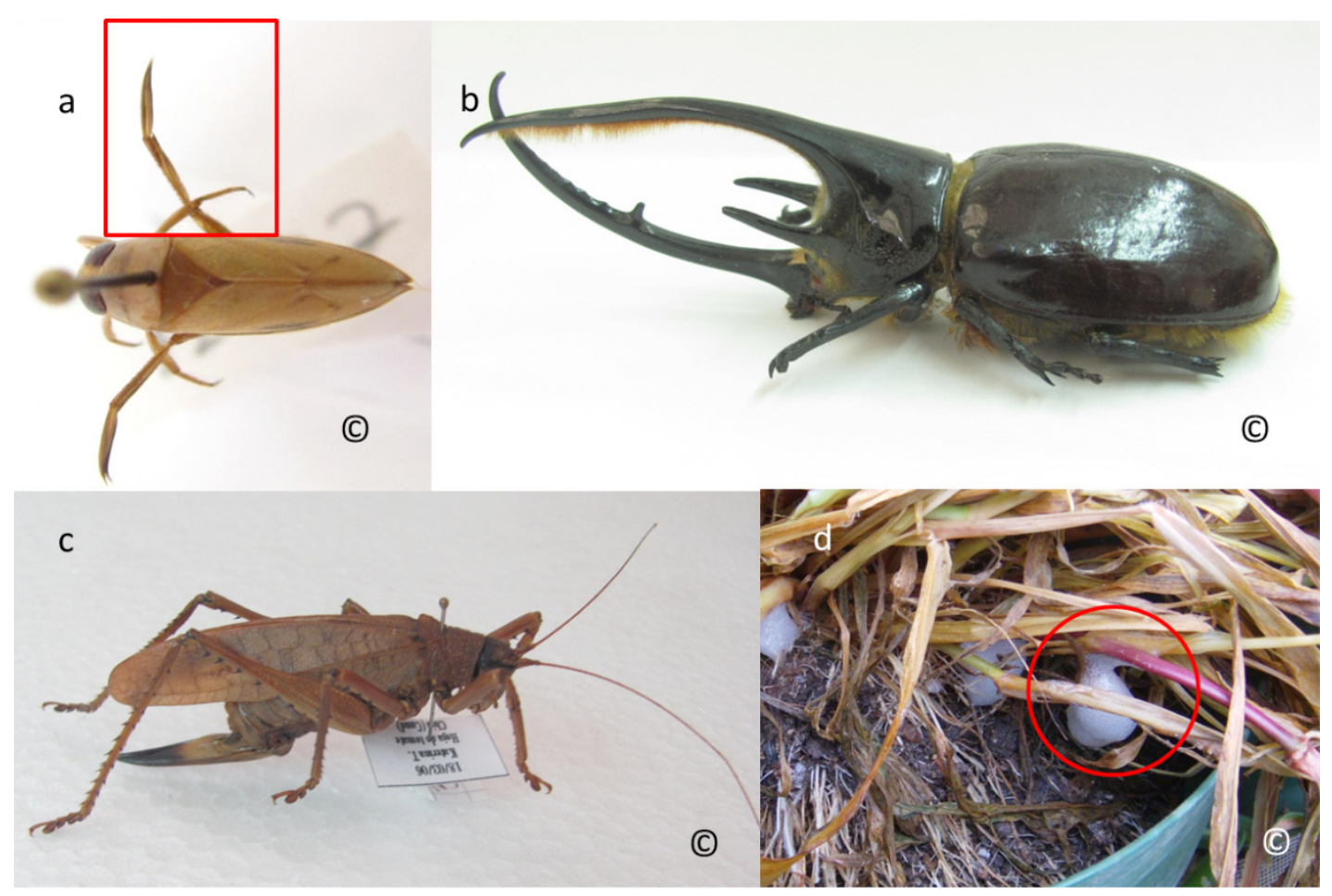

Fotografía 2: a) Notonecta sp., nótese las patas traseras que el insecto emplea como remos; b) Dynastes hercules la robustez corporal; c) Tettigonia sp., los agrandados fémures de las patas traseras facilitan el salto a este campeón de salto alto; d) Ninfas envueltas en espuma del salivazo o mión Aneolamia sp. (Fotografías a, b y c tomadas por Ing. Agr. Héctor William Duarte y d. por Laura Romero, estudiante Ing. Agronómica U.D.C.A, pasante CIAT, Palmira). 
no Aaron Peirsolcon, quien logró 52,54seg/100m, en Pekín, 2008. El insecto aunque no sería campeón mundial, alcanza una velocidad increíble para un animal tan pequeño. Si se toman la máxima distancia nadada por segundo, $60 \mathrm{~cm}$ alcanzado por el insecto, su velocidad se disminuye a $166 \mathrm{seg} / 100 \mathrm{~m}$, es decir, prácticamente, el doble del mejor nadador humano; sin embargo, cálculos en el primer caso, suponiendo una altura para el campeón Lenny de $1,80 \mathrm{~m}$, el gastaría 0,96 segundos en desplazar su propio tamaño, mientras que suponiendo una longitud del insecto de $1,5 \mathrm{~cm}$, éste tardaría 0,02 segundos en desplazar su tamaño. De acuerdo a esto, el insecto es más rápido y, si el humano tuviera la morfología del insecto, podría recorrer los 100 metros en 1,11 segundos.

De acuerdo con Padilla-Gil (1994), los machos de la especie N. melaena Kirkaldy tienen una longitud de 8,67-15,30mm y las hembras $8,53-15,00 \mathrm{~mm}$. Este estudio no distinguió entre los dos sexos, así que la velocidad que muestran los adultos corresponde a cualquiera de los dos y podrían ser comparados con estaturas de 1,72 a 1,85m, de los campeones humanos.

Triplehorn \& Johnson (2007) mencionan que estos depredadores de otros insectos son nadadores rápidos y que alcanzan grandes velocidades, moviendo sus patas traseras, como remos. Se alimentan chupando la hemolinfa de sus presas y, además, son capaces de morder a los humanos al ser manipulados, mordedura que se asemeja a la picadura de una abeja.

Pos. Neosigara es similar, en apariencia general, a Notonecta: nada "pecho" con las patas traseras alargadas y en forma de remo; su cuerpo es ovalado y se alimenta, por lo general, de algas y es presa de otros animales acuáticos. Como se puede observar en la tabla 1, en general, nada distancias más cortas en menos tiempo que la nadadora de espalda, pero muestra una mayor variación, tanto entre la distancia recorrida como en la velocidad lograda que Notonecta. Convirtiendo los datos de la tabla a un récord comparable con aquel que obtuvo el japonés Kosuke Kitajima, en el 2008, en Pekín, de 58,91seg/100m, el corixido lograría abarcar, en promedio, 100m en 132 segundos; su velocidad máxima correspondería a $89 \mathrm{seg} / 100 \mathrm{~m}$, marca que se acerca bastante al de los humanos.

Los humanos son capaces de nadar distancias considerables, mientras que ambos insectos no requieren realizar estas faenas, pues normalmente encuentran a su alimento, otros insectos y algas, relativamente cerca. Igualmente, no compiten por medallas, pero sí por presas, como es el caso de Notonecta, con otros individuos de la misma especie. Además, los humanos se someten a entrenamientos rigurosos, a menudo extenuantes, mientras que los insectos adquieren estas habilidades evolucionando para poder sobrevivir.

Atletismo:

En este deporte debemos distinguir entre las categorías de correr y de saltar alto y largo. En ambas disciplinas, los hombres son los perdedores si se enfrentaran, bajo las mismas circunstancias y características, a los insectos.

La cucaracha americana Periplaneta americana L. 1758 (Blattodea: Blattidae) ha sustentado el récord durante muchos años, en ser el corredor más rápido entre los insectos, con $1,5 \mathrm{~m} / \mathrm{s}$, velocidad medida por Full \& Tu (1991), quienes utilizaron, entre otros equipos, cámaras de alta velocidad para medir el tiempo y los movimientos a determinada distancia. Esta velocidad relativa correspondería a desplazarse $100 \mathrm{~m}$ en 66,66 segundos, si se buscara una equivalencia con los humanos, récord que es sustentado por Usain Bolt (JAM), quien corría 100m lisos, en 9s 58', es decir, aunque la cucaracha americana es muy rápida no podría competir con el hombre.

Sin embargo, Kamoun \& Hogenhout (1996) afirman que los cicindélidos del género Cicindela subgénero Rivancindela, denominados también "Australian tigerbeetles", específicamente el C. hudsoni, supera considerablemente a P. americana, pues puede correr a una velocidad de $2,49 \mathrm{~m} / \mathrm{s}$, lo que corresponde a 40,16s/100m. El género Cicindela es común en Colombia: es un insecto benéfico, ya que se alimenta de insectos plagas. Requiere, precisamente, moverse con rapidez para alcanzar sus presas.

Hasta hace menos de diez años, se solía atribuir a la pulga el hecho de ser el insecto que sustenta la marca de salto. Lyon (2007) menciona que las pulgas son excelentes saltadores, capaces de saltar verticalmente hasta $18 \mathrm{~cm}$ y horizontalmente $33 \mathrm{~cm}$, teniendo los adultos, de este insecto, una longitud que varía de solamente 0,16 a $0,32 \mathrm{~cm}$. Traduciendo estas cifras a una equivalencia para los humanos, un hombre tendría que saltar verticalmente $76 \mathrm{~m}$ y horizontalmente $137 \mathrm{~m}$, aproximadamente.

Empleando una cámara de alta velocidad, Burrows (2003) determinó que un mión o salivazo, el Phalaenus spumarius (L. 1758) (Hemiptera: Cercopidae), un insecto de apenas $6 \mathrm{~mm}$ de largo, es capaz de saltar más de $60 \mathrm{~cm}$, es decir, casi el doble del logro de la pulga; lo consigue gracias a su mayor poder de aceleración, más de 400 veces la fuerza de gravedad $(400 \mathrm{~g})$, mientras que la pulga solamente acelera 135 veces y un ser humano, aproximadamente, $5 \mathrm{~g}$. Sería interesante estudiar el salto de un mión colombiano, del género Aenolamia (Fotografía 2d), plaga de pastos, a ver si rompe el récord. En comparación con las marcas descritas, 
el hombre alcanza saltar hasta 2,45m, marca que sostiene el cubano Javier Sotomayor y en el salto de longitud 8,95m, logrado por el norteamericano Mike Powell.

Otro insecto, que de acuerdo a la literatura compite con los anteriores, es el chapulín o saltamontes, Tettigonia viridíssima (L. 1758) (Orthoptera Tettigoniidae). Al comparar su peso y su tamaño con otros insectos, éste sobresale entre los saltadores más vigorosos del reino animal y se estima que pueden saltar más de 30 veces su tamaño corporal. Se le atribuye un salto de $3 \mathrm{~m}$ de un solo brinco, es decir, si el chapulín mide $5 \mathrm{~cm}, 60$ veces su altura. Proporcionalmente, si un hombre saltara como un chapulín, se podría elevar 850m en el aire (Castillo \& Hernández. 1998). Estos datos, lamentablemente, no están soportados por estudios científicos o, por lo menos, si existen, no se encontraron en la bibliografía revisada; no obstante, las observaciones de campo acerca de saltos de los saltamontes indican saltos largos de, aproximadamente, $1 \mathrm{~m}$ de manera espontánea, al ser molestados. Sus patas traseras (Fotografía 2c), con los fémures fuertes y ensanchados, les permite realizar brincos monumentales, que dificultan ser alcanzados, ya sea por colectores de insectos o per enemigos naturales, tales como pájaros.

Levantamiento de pesas o Halterofilia:

Deporte que cobra especial importancia para Colombia, ya que es la única medalla de oro para el país, lograda por María Isabel Urrutia, en los Juegos Olímpicos de Sydney, Australia, al ganar en la categoría de $75 \mathrm{~kg}$; en su primer intento levantó $110 \mathrm{~kg}$ y, en el segundo, 135kg. ¿Podrían los insectos competir en esta disciplina, que forma parte de las Olimpiadas desde Atenas 1896 y abolida de ellas, en 1908, para ser reivindicada en Ámsterdam 1928 (I.E.S. «García Morato, s.f.), con nuestra campeona?

Al respecto, llamaron la atención las cifras, aparentemente, exageradas y contradictorias, del peso que podrían levantar algunos insectos, entre ellos las hormigas, que muestra la literatura gris. Indica Anónimo (2009), que una hormiga, pero no dice que especie o género, puede levantar un objeto que pesa cincuenta veces más que ella; esta opinión la confirma Castro (2011) y agrega que el formícido es capaz de soportar una carga 30 veces el volumen de su cuerpo. Las obreras de las hormigas arrieras, Atta spp. (Hymenoptera: Formicidae, Myrmicinae), plagas de importancia agrícola en todo América, conocidas también como culonas, zompopas o cortadoras de hoja, se distinguen por transportar en sus mandíbulas, pedazos de hoja hasta su nido. Cada hormiga puede transportar hojas que pesan de 5 a 20 veces el peso de su cuerpo; esto es como si un hombre de $100 \mathrm{~kg}$ transportara una carga de 2.000kg (Depto. de Biología y Ecología, 2009-2010).
Sin embargo, existen experimentos que buscan medir la capacidad de carga y sus implicaciones en el comportamiento de cortar hojas de las arrieras. Burd (2000) determinó que el tamaño del cuerpo de las obreras, expresado en masa corporal, afecta notoriamente la capacidad de carga de estas hormigas polimórficas. Logró estas conclusiones con obreras de Atta cephalotes de masa corporal, que varía entre 5 hasta $15 \mathrm{mg}$.

Si se extrapola esta masa corporal y se aplica a los datos del peso de hojas de un cítrico y de la planta ornamental Eugenia, analizadas en la tabla 2, se deduce que, si las obreras cargan pesos de hojas de cítricos entre 0,047 y $0,193 \mathrm{~g}$ y de Eugenia entre 0,0844 y 0,3042g, respectivamente, las obreras menos pesadas podrían cargar de 24,2 a 33,5 veces su peso corporal (basado en las medias). Las obreras más pesadas podrían cargar, entonces, solamente, entre 8,06 y 11,16 veces su masa corporal; no obstante, si se toman los máximos pesos de las hojas, la carga de las obreras menores correspondería a 38,6 veces su peso en pedazos de hojas de cítricos y 60,84 veces, si la carga correspondería a pedazos de Eugenia.

Tabla 2. Estadísticas descriptivas del peso (en gramos) de los pedazos de follaje, que podrían cargar las obreras de Atta sp.

\begin{tabular}{|l|l|l|}
\hline & Eugenia & Media \\
\hline Cítrico & 0,120977273 & 0,16742444 \\
\hline Desviación estándar & 0,030823193 & 0,0486887 \\
\hline Rango & 0,2198 & \\
\hline Mínimo & 0,047 & 0,0844 \\
\hline Máximo & 0,193 & 0,3042 \\
\hline $\begin{array}{l}\text { Nivel de confianza } \\
(95,0 \%)\end{array}$ & 0,009371107 & 0,0146277 \\
\hline
\end{tabular}

Datos muy diferentes fueron estimados por Tonhasca \& Lima Bragança (2000) para A. cephalotes, al comparar la masa corporal de las forrajeras $7,3 \pm 4,1 \mathrm{mg}$ con la masa de los fragmentos de hoja 21,9mg; los autores, en general, concluyeron que un aumento en la masa corporal resulta en una más alta capacidad de carga. Teniendo en cuenta estas cifras, las obreras de esta arriera solamente podrían cargar tres veces su peso, así que la incógnita sobre su fuerza real sigue en pie.

Compite con las hormigas el cucarrón rinoceronte, Dynastes hercules (L.). (Coleoptera: Melolonthidae: Dynastinae) (Fotografía 2b), que en el país tiene una amplia distribución geográfica y ha siso colectado en los departamentos de Amazonas, Antioquia, Boyacá, Casanare, Chocó, Cun- 
dinamarca, Meta, Nariño, Putumayo, Santander y Valle. De acuerdo a Animal Corner (2012) es el más fuerte entre los animales vivientes del planeta y se le atribuye la capacidad de levantar hasta 850 veces su propio peso, equivalente a que si un ser humano de peso y altura promedio tuviese la fuerza de levantar 65t, peso inimaginable para un hombre. Experimentos con este insecto realizados por R. Kram, fisiólogo de la Universidad de California, Berkely, mostraron que aunque los cucarrones eran capaces de cargar 100 veces su propio peso, con esta carga casi no eran capaces de moverse. Concluyó el investigador que una carga justa corresponde a 50 veces el peso del $D$. hercules (Zimmer, 1996).

El récord en levantamiento de pesas lo sostiene; sin embargo, otro coleóptero, el Onthophagus taurus (Coleoptera: Scarbaeidae, Coprinae), comúnmente denominado mierdero o pelotero, el escarabajo sagrado de la cultura egipcia, se le atribuye la capacidad de levantar hasta 1.141 veces su propio peso. El Onthophagus taurus (2010) debería ser entonces apto para levantar 102,69t. El record olímpico obtenido por Hossein Rezazadeh, en Sydney, 2000, para hombres de más de $105 \mathrm{~kg}$ - arrancada - fue de solamente $212,5 \mathrm{~kg}$, escasamente el doble del peso corporal del competidor; nuevamente, ganaría un insecto si estaría compitiendo bajo condiciones apropiadas para el ser humano y el artrópodo.
Lucha libre:

El combate entre insectos, ante todo para defender el territorio, para ocupar áreas de interés o para asegurar alimento, es muy frecuente entre los insectos, especialmente entre hormigas, ya sea de la misma especie o de otras especies, inclusive, subfamilias diferentes. Un caso de lucha a muerte, no comparable con la lucha libre que se realiza en olimpíadas específicas, todavía no incluidas en las oficiales, se detalla, brevemente (Zenner de Polanía, 2012). Aparentemente por territorio, ocurrió la pelea entre representantes de dos subfamilias de la familia Formicidae (Hymenoptera), la primera más pequeña, Nomamyrmex (Ecitoninae) y, la otra, Camponotus sp. (Formicinae) de color miel, de tamaño mayor (Fotografía 3). Contrario a los humanos, las hormigas usaron sus mandíbulas como armas. La obreras de Camponotus huían, perseguidas por las otras, consideradas las más agresivas. La lucha, en la cual participaban las castas obreras de las dos especies, terminaba al quedar muerta, por lo general, Camponotus. ¿Se podría comparar este combate con la lucha a muerte de los gladiadores romanos?

Basado en todas las comparaciones anteriores, queda sobre el tapete la pregunta: ¿serán los humanos capaces de seguir rompiendo los récords olímpicos en el futuro? No se acercarán a algunos sostenidos por insectos, lo que se po-

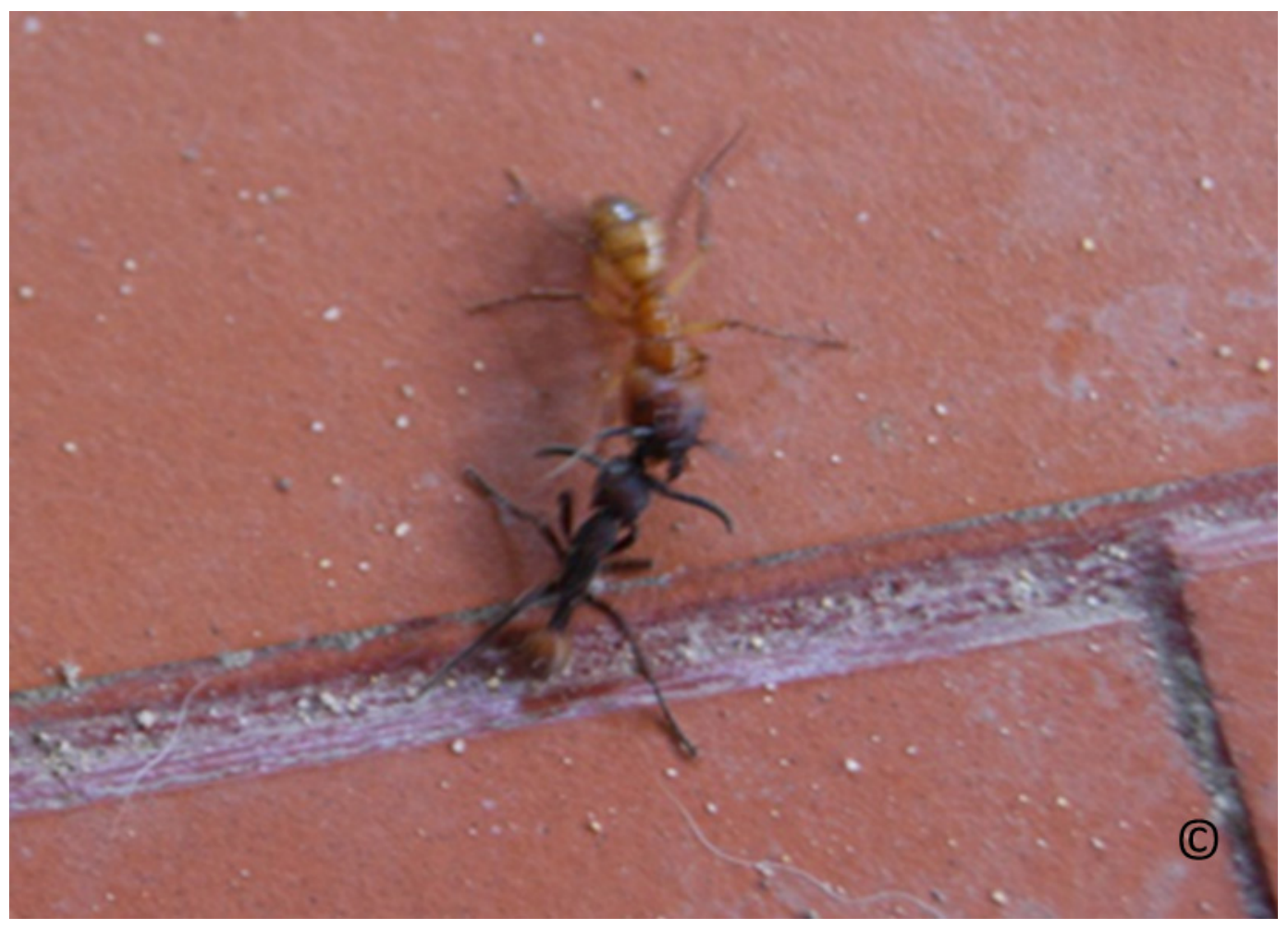

Fotografía 3. Lucha a muerte Camponotus vs. Nomamyrmex (tomada por la autora). 
dría atribuir a que estos últimos les llevan millones de años de ventaja en su evolución. Se concluye, además, que muy probablemente existen muchos otros insectos que podría ser campeones olímpicos si compitiesen con el hombre, pero se desconocen sus habilidades "deportivas", haciendo falta investigaciones al respecto.

Agradecimientos: Al Ingeniero Agrónomo Helber A. Arévalo Maldonado, por su colaboración en los análisis estadísticos; a Héctor William Duarte Gómez por la toma de fotografías. Conflicto de intereses: El autor declara que no existe ningún conflicto de intereses que ponga en riesgo la validez de los resultados presentados.

\section{BIBLIOGRAFÍA}

1. ANIMAL CORNER. 2012. Rhinoceros Beetle. Disponible desde Internet en: http://www.animalcorner. co.uk/insects/beetles/beetle_rhino.html (con acceso 25/01/12).

2. ANÓNIMO. 1997. Información de la Mantis religiosa. Disponible desde Internet en: http://insected.arizona. edu/espanol/mantidinfo.htm (con acceso 14/11/10).

3. ANÓNIMO. 2009. Datos curiosos sobre insectos. Disponible desde Internet en: http://wweespanol.forumcommunity.net/?t=23084394 (con acceso 09/12/11).

4 ARMITAGE, M.H.; MULLISEN, L. 2003. Preliminary Observations of the Pygidial Gland of the Bombardier Beetle, Brachinus sp. Disponible desde Internet en: http://www.answersingenesis.org/articles/tj/v17/n1/ beetle (con acceso 09/11/11).

5 ARNEDO, J.L. s.f. Tanglangquan o boxeo de la mantis. Disponible desde Internet en: http://www.wushulonghuquan.com.ar/Tanglangquan\%20Mantis.pdf (con acceso 30/11/11).

6. BURD M. 2000. Body size effects on locomotion and load carriage in the highly polymorphic leaf-cutting ants Atta colombica and Atta cephalotes. Behav. Ecol. 11:125-131.

7 BURROWS, M. 2003. Biomechanics: froghopper insects leap to new heights. Nature. 424:509.

8. CASTILLO C., C.; HERNÁNDEZ F., V. 1998. Chapulín. Disponible desde Internet en: http://redescolar.ilce. edu.mx/redescolar/publicaciones/publi_reinos/fauna/chapulin/chapulin1.htm (con acceso 19/12/11).

9. CASTRO, B. 2011. El mundo de las hormigas. Disponible desde Internet en: http://www.slideshare.net/brigittecastro/hormigas-7635357 con acceso 19/12/11).

10. DEAN, J.; ANESHANSLEY, D.J.; EDGERTON, H.E.; EISNER, T. 1990. Defensive spray of the Bombadier Beetle: a biological pulse jet, Science. 248:12191221.

11. DEPTO DE BIOLOGÍA Y ECOLOGÍA. 2009-2010. Ciencias de la naturaleza. Disponible desde Internet en: www.educa.madrid.org/web/ies.atenea. sansebastian/.../1eso.html (con acceso 21/11/11).

12. EISNER, T.; ANESHANSLEY, D.J.; EISNER, M.; ATTYGALLE, A.B.; ALSOP, D.W.; MEINWALD, J. 2000. Spray mechanism of the most primitive Bombardier Beetle (Metrius contractus). J. Exp. Biol. 203:12651275.

13. ENGEL, M.; GRIMALDI, D. 2004. New light shed on the oldest insect. Nature. 427:627-630.

14. FULL, R.J.; TU, M.S. 1991. Mechanics of a rapid running insect: Two, four and six-legged locomotion. J. Exp. Biol. 156:215-231.

15. I.E.S. "GARCÍA MORATO". s.f. Los juegos olímpicos en la actualidad. Disponible desde Internet en:http://iesgarciamorato.org/Dep_Griego/juegos/trabajos/pruebas_actuales.htm (con acceso 18/11/11).

16. KAMOUN, S.; HOGENHOUT, S.A. 1996. Flightless and rapid terrestrial locomotion in tiger beetles of the $\mathrm{Ci}$ cindela L. subgenus Rivacindela van Nidek from saline habitats of Australia (Coleoptera: Cicindelidae). Coleopt. Bull. 50:221-230.

17. LYON, W.F. 2007. Fleas. Ohio State University Extension Fact Sheet. Entomology. 9p. Disponible desde Internet en: http://ohioline.osu.edu/hyg-fact/2000/2081. html (con acceso 09/12/11).

18. MARCA (DEPORTE). 2009. Récords mundiales y olímpicos. Disponible desde Internet en http:// es.wikipedia.org/wiki/Marca_(deporte)\#R. C3.A9cords_mundiales_y_ol.C3.ADmpicos (con acceso 09/01/12). 
19. McCOMB, D.G. 1999. Sports: An Illustrated History. Oxford University Press. 144p.

20. NATACIÓN. 2008. Records. Disponible desde Internet en: http://archivo.marca.com/otrosdeportes/natacion/records/distancia larga masculino.html (con acceso 09/01/12).

21. Onthophagus taurus. 2010. Onthophagus taurus el insecto más fuerte del mundo. Disponible desde Internet en: http:/www.maikelnai.es/2010/03/25/ onthophagus-taurus-el-insecto-mas-fuerte-delmundo/ (con acceso 19/01/12).

22. PADILLA GIL, D.N. 1994. Bioecología y sistemática de Notonecta melaena Kirkaldy (Hemiptera, Notonectidae) en Cundinamarca-Colombia. Agr. Col. 11(1):34-52.

23. RECORDS OLÍMPICOS. 2004. Halterofilia. Disponible desde Internet en: http://olimpia.galeon.com/verano/ records/records.htm (con acceso 09/01/12).

24. SOFTPEDIA. 2011. The-Technology-of-the-Bomabardier-Beetle-Has-Been-Explained. http://news.softpedia.com/news/The-Technology-of-the-Bomabardier-Beetle-Has-Been-Explained-82268.shtml (con acceso 14/01/12).

25. TALADOIRE, E. 2000. El juego de pelota mesoamericano. Origen y desarrollo. Rev. Arqueol. Mexicana. 8(44):20-27.
26. TONHASCA, A.; LIMA BRAGANÇA, M.A. 2000. Forager size of the leaf-cutting ant Atta sexdens (Hymenoptera: Formicidae) in a mature eucalyptus forest in Brazil. Rev. Biol. Trop. 48(4):983-988.

27. TRIPLEHORN, C.; JOHNSON, F. 2007. Borror's Introduction to the Study of Insects. $7^{\text {th }}$ ed. Edit. Thomson Learning ${ }^{\mathrm{TM}}$. p.290-291.

28. URIARTE, M.T. 2000. Mariposas, sapos, jaguares y estrellas. Práctica y símbolos del juego de pelota. Rev. Arqueol. Mexicana. 8(44):28-35.

29. WIKILIBROS. s.f. Lanzamiento de jabalina. Disponible desde Internet en: http://www.deportes.gov.co/ lanzamiento-de-jabalina-historia.html (con acceso 14/01/2012).

30. ZENNER DE POLANÍA, I. 2012. Pelea a muerte. Notas y Noticias Entomológicas. Universidad de Ciencias Aplicadas y Ambientales U.D.C.A. Bol. Inf. (online) 32(4).

31. ZIMMER, C. 1996. Beetle of Burden. Disponible desde Internet en: http:/dicovermagazine.com/1996/apr/ beetle of burden744 (con acceso 09/01/12).

Recibido: Enero 16 de 2012

Aceptado: Abril 11 de 2012 